\title{
Protée
}

\section{Le corps : anatomie d'un symbole}

\section{Paola Pacifici}

Volume 36, numéro 1, printemps 2008

Le symbole. Réflexions théoriques et enjeux contemporains

URI : https://id.erudit.org/iderudit/018803ar

DOI : https://doi.org/10.7202/018803ar

Aller au sommaire du numéro

Éditeur(s)

Département des arts et lettres - Université du Québec à Chicoutimi

ISSN

0300-3523 (imprimé)

1708-2307 (numérique)

Découvrir la revue

Citer cet article

Pacifici, P. (2008). Le corps : anatomie d’un symbole. Protée, 36(1), 29-38.

https://doi.org/10.7202/018803ar

\section{Résumé de l'article}

Grâce à la dissection, une idée nouvelle du corps s'affirme à l'aube de la Renaissance : il s'agit d'un corps matériel et saisissable, qui trouve dans son nouveau statut les prémisses - philosophiques et esthétiques - de sa représentation. Néanmoins, loin de se réduire à un schéma fonctionnel, le corps anatomique se détermine dans le cadre d'une saisie symbolique qui le lie étroitement à la religion, à l'astronomie et à l'art. Par l'analyse d'un choix de textes, cet article se propose de revoir cette construction à la fois symbolique et scientifique et d'expliquer comment le corps se définit dans un réseau pragmatique, devenant une clefd'interprétation du monde. 


\section{LE CORPS : ANATOMIE D'UN SYMBOLE}

PAOLA PACIFICI

Un corps nouveau s'affirme à la Renaissance, lorsque la dissection devient l'outil principal du savoir anatomique. Son image se définit, à cette même époque, comme une construction «vraie» de la réalité, perspective et mathématique.

Le corps est analysé dans sa structure intérieure par le biais d'un regard qui force ses confins et construit un nouvel espace de pertinence pour la définition de sa visibilité. Ce qui était caché, enfoui dans la profondeur anatomique et auparavant considéré comme matériellement insaisissable, devient l'objet d'une réflexion à la fois philosophique et scientifique qui passe par la notion de forme. La fragmentation anatomique devient le matériel expressif d'un discours et d'une image qui affirment l'existence du concept même de corps, et cela dans l'énonciation d'une double problématique: l'affirmation du corps en tant que réalité sensible et de la possibilité de décrire visuellement sa structure anatomique. L'image scientifique du corps que l'anatomie met en place au début de la Modernité se définit comme objective grâce à l'emploi de stratégies de sens qui affirment la véracité du corps représenté. La physique des organes trouve sa définition dans le cadre d'une métaphysique du cadavre, texte expérimental et espace artificiel apte à la saisie d'un code d'interprétation. L'établissement d'une «relation de renvoi», au sens de Peirce, entre la chair et le concept, passe par une articulation sémiotique - et plus précisément symbolique - entre texte, code et réalité.

Le corps anatomique - un ensemble organisé de parties qui fonctionnent selon des règles internes - garde, intacte et fonctionnelle, la mémoire de ce qu'il fut avant d'être soumis à la dissection: une réalité construite sur un modèle discursif et textuel. La relation qui s'instaure entre le corps réel, les textes qui le décrivent et le code qui permet de les lier n'est donc pas simplement descriptive, mais se construit comme un véritable renvoi sémiotique - relation qui caractérise le signe en tant que tel. Le corps est alors, en général, «signe», en ce qu'il participe de la semiosis illimitée qui permet au sens de se donner et de se traduire, et, en particulier, «symbole», en ce qu'il se base sur l'articulation entre un référent et une signification de nature sociale et conventionnelle. Ce type de fonctionnement 
permet de comprendre comment le corps s'affirme, à la fois en tant que réalité matérielle et réseau symbolique; sa polysémie trouve ainsi sa justification dans la dimension sociale et conventionnelle où le corps anatomique existe et "fait sens".

D'un point de vue théorique, ces considérations montrent bien les implications pragmatiques de l'approche sémiotique peircienne et leur importance dans le cadre de notre réflexion. Ainsi que l'affirme Benveniste, la communication est une affaire sociale et symbolique; plus que la caractérisation du symbole en relation avec les autres catégories de signes, il nous importe ici de montrer la pertinence de la conception pragmatique du «fonctionnement symbolique» qui est à la fois principe et pouvoir formateur des concepts et de la réalité. Cette conception nous permet en effet de considérer sur un même plan signes et réalités, et de réfléchir au corps non comme à un simple référent réel du discours anatomique, mais bien comme à une construction symbolique articulée et créatrice de nouveau savoir. Ainsi, l'individuation d'une séméiotique du corps implique une hypothèse plus généralement sémiotique sur la possibilité d'interpréter le corps en tant qu'objet doué de sens: c'est en ce sens qu'il devient signe et fonctionne de manière symbolique.

Il s'agira d'abord d'interroger l'objectivité de ce corps anatomique, qui trouve sa pleine visibilité à la Renaissance, pour s'attacher ensuite à en décrire le fonctionnement symbolique.

La réalité anatomique "prend corps», il convient de le rappeler, dans un parcours qui s'accomplit par voie discursive et textuelle. Le savoir anatomique se développe, jusqu'au XIVe siècle, par le seul biais d'une parole - constamment travaillée dans sa forme et ses contenus - qui théorise et construit la notion de corps anatomique en deçà de toute exigence de représentation visuelle. Le discours traditionnel façonne ainsi la saisie conceptuelle du corps depuis Hippocrate qui, associant des noms à sa structure et à son fonctionnement, rend l'anatomie objet d'une semiosis. Les écrits physiques d'Aristote définissent ensuite les limites d'un corps physique envisageable par analogie avec le corps des animaux déjà soumis à l'observation directe. Ce déplacement de sens, métaphorique, donne lieu à un savoir se construisant en deçà de son référent propre: un savoir analogique. Aucune observation directe n'est accomplie sur le corps humain, exception faite des travaux d'Hérophile et Érasistrate. Galien fournit une synthèse des savoirs acquis fonctionnant comme un modèle pour les interprétations à venir. Un paradigme de signification se construit sur l'invisibilité imposée par les limites infranchissables du corps désormais envisageable comme un espace en profondeur construit sur un double espace de pertinence: l'extérieur et l'intérieur. Du Ve siècle avant J.-C. à la fin du XIII' siècle, le corps est en somme une affaire de parole.

L'épaisseur anatomique du corps s'affirme, par sa description théorique, en tant qu'espace signifiant, tout en demeurant insaisissable dans sa matérialité. Le corps ne devient l'objet d'une observation directe qu'à la fin du Moyen Âge, lorsque se généralise la

dissection. Signifiant de plusieurs signifiés, le corps affirme son caractère polysémique et métaphorique. L'image devient métaphore du texte: elle est la surface qui illustre une profondeur et l'extériorisation d'un intérieur. Produit du regard analytique qui informe la dissection, l'illustration se fait ainsi porteuse de l'apparence du corps "ouvert» au sens. La rupture imposée à la chair marque un nouveau régime de visibilité: l'image recueille la mise à nu de l'anatomie et des schémas qui la rendent visible.

L'anatomie s'affirme comme un espace liminaire au croisement de plusieurs savoirs: la traduction, la paraphrase et les commentaires auxquels le savoir textuel est soumis donnent à l'image un champ d'existence élargi, touchant, outre à la médecine, à l'astrologie, à la religion, à la philosophie et à l'art. Ce traitement métaphorique du savoir sur le corps étend le champ de sa définition, en même temps qu'il précise les questions que chaque discipline peut - et doit - lui poser: sa construction en image est donc non pas la simple conséquence d'un savoir désormais accessible grâce à la dissection, mais bien le résultat d'un travail conjoint que l'anatomie accomplit en 
collaboration avec d'autres disciplines qui envisagent le corps comme un réseau symbolique.

Le nouveau statut épistémologique que le corps acquiert au début de l'époque moderne se construit sur le modèle de l'exégèse textuelle et permet, en cela, d'accomplir quelques réflexions préliminaires au sujet $\mathrm{du}$ fonctionnement symbolique sur lequel le discours scientifique est fondé. Comme Umberto Galimberti l'explique, ce discours se trouve lié davantage au jeu référentiel des textes théoriques qu'à un nouveau rapport de référence du signe au réel.

La valeur référentielle est annulée en faveur $d u$ jeu structurel $d u$ discours scientifique qui rend compte des choses choisies et identifiées pour lui obéir. Sans plus tenir lieu de quelque chose de réel, car chaque référent s'est volatilisé dans l'«objectivité» gagnée, tous les signes dans lesquels la science s'exprime peuvent s'échanger dans un libre jeu structurel, libre aussi du poids que le corps et le monde de la vie portent avec eux.

(Galimberti, 1999: 48; notre traduction)

Le corps anatomique est donc une construction théorique plutôt que la découverte d'une structure objective que la dissection révélerait dans sa matérialité. La connaissance du corps devient nécessaire puisqu'elle donne accès à un réseau de savoirs qui dépassent ceux de l'anatomie. L'entrelacement des savoirs qui trouvent application dans l'étude du corps suggère, par ailleurs, la consubstantialité des enjeux scientifiques, philosophiques et artistiques ainsi que l'opportunité de les considérer comme les facettes complémentaires d'une approche symbolique du corps.

La saisie scientifique du corps, telle qu'elle commence à prendre forme à la fin du Moyen Âge, s'accompagne d'une réflexion sur la place de l'homme dans le monde et sur sa relation avec la Création. L'astrologie et la religion, en tant qu'univers de croyance liés à la construction d'un savoir scientifique, ont en ce sens un poids considérable dans le développement d'une attitude empirique face au corps, et plus généralement dans la mise en place d'une attitude de curiosité et de confiance dans l'aspect physique du corps et du cadavre. Le corps acquiert ainsi des valences symboliques, qui vont forger des métaphores dans le but de concevoir le corps humain comme "microcosme", lieu d'union entre les différentes sphères du savoir et du monde. L'idée ancienne de l'homme-microcosme telle qu'elle se développe entre les IX ${ }^{\mathrm{e}}$ et XII ${ }^{\mathrm{e}}$ siècles, façonne l'idée d'une anatomie symbolique recelant la complexité de la création divine. L'homme, selon Jean Scot Érigène (Periphyseon, III, 37, P.L. 122, 733 B) vit comme une plante, sent comme un animal et raisonne selon les lois de l'intellect divin, idée reprise par Albert le Grand (Somme de théologie, II, 61). Robert Grosseteste, dans son traité Quod homo sit minor mundus, s'appuie également sur l'idée commune de la partition du corps en éléments naturels et compare les organes à des parties de l'univers: la tête représente le «ciel» de l'homme, et les yeux constituent son soleil et sa lune. L'ouvrage de Bernard Silvestre, De mundi universitate, sive Megacosmus et Microcosmus, publié autour de 1145, montre la manière de voir et d'inventer le corps qui se développe au XII siècle. Ce texte établit des corrélations entre les éléments et les humeurs, les astres et les propriétés organiques (en particulier, II, 13) en établissant une hiérarchie de fonctions qui rend pensables l'homme et l'univers dans le cadre de la Création.

La médecine du Moyen Âge se construit ainsi, avant tout, comme un imposant système symbolique, s'appuyant sur un réseau de correspondances étroites et complexes entre science, astrologie et théologie. Signalons que les "préjugés métaphysiques» (Nardi, 1958: 72) qui habitent le corps à l'aube de sa saisie scientifique représenteront, à nos yeux, non pas l'égarement irrationnel d'une société encore éloignée de notre conception moderne de la science, mais bien une étape importante de l'objectivation de la chair, qui constitue une prémisse nécessaire pour la saisie expérimentale du corps. Les écrits médicaux et philosophiques de l'Antiquité, redécouverts grâce à la circulation des traductions, sont interprétés à la lumière de la croyance, philosophique et religieuse, en l'harmonie parfaite de l'univers régi par les lois mêmes qui président à l'organisation du corps 
humain. Le corps est traduit en un système mathématique compatible avec la théologie et devient, en ce sens, une métaphore du monde. Le développement parallèle du corps en tant que réalité physique et de son traitement symbolique en tant que question théologique forgera un imaginaire multiforme, touchant à plusieurs champs d'investigations.

Ces représentations, conçues sous l'influence des idées néoplatoniciennes, donnent au corps un champ d'existence élargi, participant de l'essence divine et insaisissable par les sens comme l'était l'Idée platonicienne. Ainsi que le rappelle Aaron J. Gourevitch:

Le symbolisme chrétien "doublait» le monde et donnait à l'espace une dimension nouvelle, supplémentaire, invisible pour les yeux, mais saisissable à travers toute une série d'interprétations. (1983: 87)

Le corps est, dans sa matérialité, un système symbolique soumis à la quête d'une interprétation; il se fait le lieu des récits de la sainteté et du martyre et devient en ce sens «une composante de plus en plus importante du discours théologique» (Nutton, 2003: 175). La Legenda Aurea de Jacques de Voragine, écrite entre 1261 et 1266, raconte, avec profusion de détails, le destin cruel des saints et contribue à la saisie chrétienne du corps et de ses fragments, comme un lieu de haute signifiance.

Le corps des saints devient en effet un objet concret d'analyse et d'interprétation, qui se développe autour des images des martyrs et du culte des reliques. Véritables parties anatomiques, elles sont, selon l'expression de David Le Breton, des «métonymie[s] de la Gloire de Dieu» (2003: 36). Piero Camporesi souligne à ce propos le développement d'une «familiarité avec l'aspect physique de la mort aujourd'hui presque incompréhensible» (1994: 10; notre traduction) qui porte à l'ouverture, voire à la "fouille» anatomique, dans un contexte différent de celui de la saisie médicale. Il évoque, à ce sujet, la dissection accomplie sur les dépouilles de Chiara da Montefalco, morte en odeur de sainteté en 1308. Les sœurs augustiniennes de son couvent, jugeant indécent de confier le corps vierge de la sainte aux soins d'un barbier en vue de son embaumement, décident d'accomplir elles-mêmes cette tâche. Après avoir prélevé les organes intérieurs, elles se rappellent les dernières paroles de la sainte: «Io tengo Giesù Cristo Crocifisso dentro il cuor mio» (je porte dans mon cour Jésus Christ crucifié [Piervirgilii, 1663; cité par Camporesi, 1994: 6]); elles procèdent alors à l'ouverture du cœur et y trouvent la figure de la croix, formée de chair, et le fouet, objet du martyre du Christ. La chronique raconte ensuite un examen supplémentaire accompli par l'évêque Berengario Donadei, qui finit par trouver dans le cour de la sainte, outre la croix et le fouet, les autres instruments de la Passion: la colonne, la couronne d'épines, les trois clous, la canne avec l'éponge et la lance si bien formée qu'elle pique le doigt de l'évêque. Cet épisode montre bien l'efficacité symbolique du corps et des métaphores qui informent sa vision et, plus généralement, le poids de la croyance dans la perception du corps.

Les études anatomiques se développent au sein d'une vision symbolique du corps. David Le Breton souligne que le corps est envisageable, au Moyen Âge, non en tant que catégorie du sujet - ainsi qu'il sera pensé par la modernité -, mais en tant que constituant du système symbolique du monde.

Les frontières de la chair ne démarquent pas les limites de la monade individuelle. Un tissu de correspondances mêle sous une destinée commune les animaux, les plantes, l'homme et le monde invisible. Tout est relié, tout résonne ensemble, rien n'est indifférent, tout évènement fait signe [...] il n'y a aucune rupture qualitative entre la chair de l'homme et la chair du monde.

(2003: 33)

Le corps anatomique ne cesse de renvoyer à sa polysémie, ainsi qu'en témoignent les images de l'homme zodiaque qui représentent le corps humain parfois les organes - en relation avec les signes zodiacaux. L'astrologie est reliée à certaines opérations cliniques comme la saignée et la phlébotomie et conquiert ainsi une utilité pragmatique. Plusieurs 
illustrations, à partir du XIVe siècle, montrent des figures zodiacales, en spécifiant leur zone somatique d'influence ainsi que les points de la saignée en relation avec un moment astrologique précis. L'anatomie «naturelle» du corps se limite à sa surface et se réduit à un schéma occupé par l'écriture ou par l'indication du signe astrologique. Ces illustrations survivent à l'ouverture anatomique du corps, ainsi que le montrent plusieurs images analogues des XVe et $\mathrm{XVI}$ e siècles, qui reprennent la disposition et la symbolique des signes zodiacaux. Le Fasciculo de medecina de Johannes de Ketham, une anthologie de textes médicaux publiée pour la première fois à Venise en 1491, reproduit le même schéma parmi ses rares illustrations. L'«homme astrologique» est considéré comme une partie du savoir médical qui, tout en donnant une signification symbolique du corps, exerce une influence concrète dans son approche clinique et n'empêche pas, comme dans le cas du Fasciculo de medecina, l'affirmation d'un savoir anatomique basé sur la dissection humaine.

L'analyse de l'anatomie par le zodiaque est un système interprétatif qui sélectionne, dans un monde où tout fait signe, deux sources de signifiance - le corps et les astres - comparées sur la base d'un fonctionnement analogue postulé à titre de critère de pertinence. La nature symbolique de leur relation, tout comme la pertinence de leur comparaison, ne fait pas l'objet d'un questionnement, car sa "vérité» n'est pas à prouver. Le système des humeurs, tel qu'il se développe dans chaque individu, est en partie influencé par la position des astres au moment de la naissance: ainsi, l'astrologie détermine, en reliant le corps à l'univers qui l'entoure, le tempérament de l'individu (colérique, flegmatique, mélancolique ou sanguin) et démontre que tout fonctionnement «somatique» est d'emblée "sémiotique».

Le système métaphorique hérité de l'Antiquité qui partageait le corps dans la triade caput/venter/membra (tête, entrailles, membres) s'enrichit d'un apparat anatomique et symbolique qui définit le rôle de chacun des organes principaux. L'imaginaire lié au cœur, siège des passions et de la spiritualité, prolifère

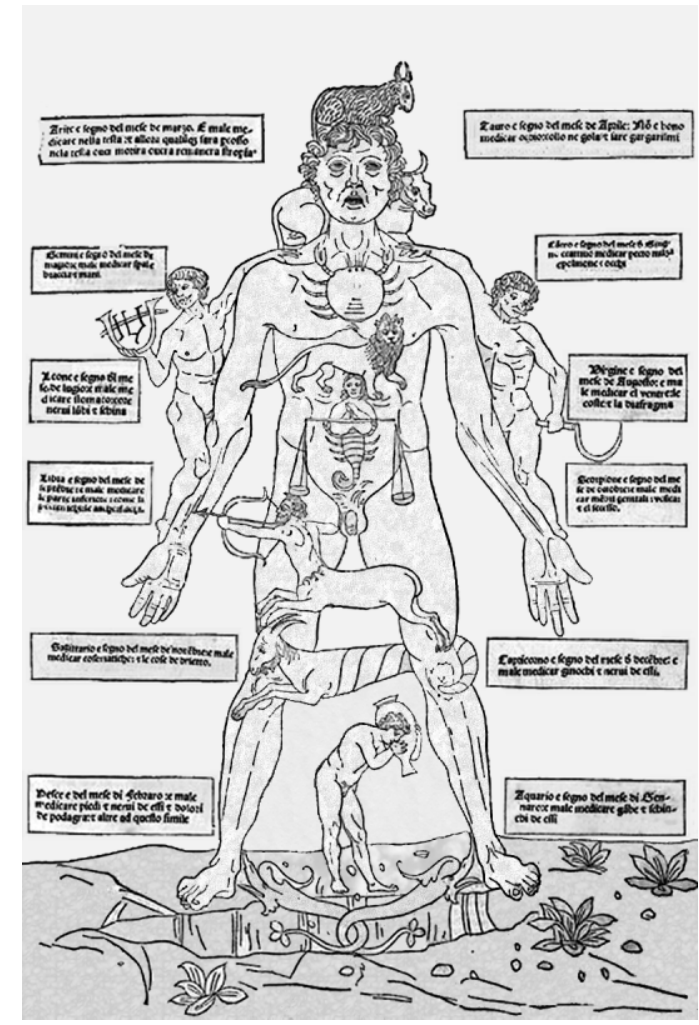

"Homme zodiaque"

Johannes de Ketham, Fasciculo de medicina, 1493.

entre les XIII et XIVe siècles; en témoigne la présence du thème du «cœur mangé» dans les récits érotiques et courtois de la littérature française de l'époque ${ }^{1}$. La tête, siège de l'âme, qui occupe la partie haute du corps, est valorisée dans sa fonction dirigeante et opposée au bas selon le principe hiérarchique. Le foie, qu'Isidore de Séville identifie avec le siège du plaisir et de la concupiscence, est relégué à la zone «basse» du corps. L'étymologie du mot «foie» - le nom archaïque de ce viscère remonte au latin: iecur, iecoris, de jacio (jeter, envoyer) - permet à l'auteur de dériver sa fonction: le foie est le lieu d'où se répand le "feu", à la fois élément formateur du corps et principe de son fonctionnement, qui va au cerveau et se diffuse dans les membres. Cette correspondance entre l'objet de référence (le foie), le mot qui le désigne et ses caractéristiques propres, témoigne du mécanisme de formation de telles métaphores et de leur efficacité explicative. 
Le «diagnostic astrologique» s'inscrit dans ce même contexte symbolique, où, selon la formule de Jacques Le Goff, «le corps en soi n'existe pas» (2003: 127). Sous les auspices favorables de l'autorité galénique, l'astrologie devient partie intégrante des connaissances médicales, qui, pourtant, semblent impuissantes face aux fléaux qui ravagent l'Europe. Le médecin universitaire, grâce à son passage préliminaire par le cursus ès arts, reçoit les bases de l'astrologie qu'il applique au fonctionnement anatomique en accord avec les textes. L'utilisation de l'astrologie, bien qu'elle s'accompagne parfois d'un retour aux croyances de la magie naturelle, anticipe sous certains aspects l'importance croissante de la pratique. En se servant d'instruments, tels que l'astrolabe pour le calcul de la position des astres, les médecins, motivés dans la croyance de l'influence des astres sur le corps de l'homme, se trouvent confrontés à des questions de technique et de mesure. Aussi développent-ils un intérêt nouveau pour l'observation et la production d'instruments dont ils sont souvent les inventeurs (Jacquart, 1995: 206) ${ }^{2}$.

Le corps, entre les Xe et XIII siècles, devient le centre d'un réseau symbolique complexe qui le relie à l'univers épistémologique et philosophique de l'époque, sans pour autant cesser de faire l'objet d'une quête rationnelle et scientifique sur sa nature. La naissance de l'université favorise les échanges interdisciplinaires et la médecine se construit en relation étroite avec la philosophie naturelle, dont elle partage l'orientation analytique. La question méthodologique qui entre dans l'interprétation des textes des autorités, dont le corpus s'agrandit grâce aux traductions, contribue à cerner un nouveau rapport entre la recherche savante et l'expérience, et à valoriser la pratique anatomique. La médecine du Moyen Âge prépare le cadre de la saisie sensorielle du corps, tout en se faisant porteuse d'un code de correspondances symboliques transcendant les cadres d'une approche scientifique. Le corps acquiert un statut de métaphore: clef d'interprétation des profondeurs du cosmos et de l'âme en même temps qu'objet du monde, il se prête au déplacement de sens propre à la semiosis et se constitue ainsi dans les interrelations de ses valences symboliques.

L'anatomie, investie de ce réseau de correspondances, devient un objet d'étude privilégié, car elle ouvre la voie à une compréhension de la structure du monde. "L'atroce envie d'étudier» (Cocchi, 1745; repris dans Camporesi, 1991: 110) des premiers anatomistes permet ainsi l'ouverture $\mathrm{du}$ corps qui est, selon les mots de Bernard Silvestre, un «second univers». Les symbolisations graphiques dont le corps fait l'objet sont conçues de manière non seulement à rappeler, au moment de leur interprétation, des significations spécifiques, mais aussi à en écarter d'autres. Il convient de «maîtriser» la polysémie du corps afin de sélectionner les contenus à transmettre: ce processus délimite le programme narratif propre aux images. Les associations métaphoriques qui informent la pensée anatomique de la Renaissance ne vont perdre leur prégnance qu'entre les XVIII e et XIXe siècles, lorsque l'anatomie trouvera sa pleine justification dans le cadre de la méthode anatomo-clinique. Chez Vésale (1514-1564) et ses contemporains, bien au contraire, le discours anatomique dépend des autres savoirs dont le corps fait l'objet - Descartes n'a pas encore établi sa dichotomie entre l'âme et le corps. L'âme est, à l'époque de Vésale, un principe immanent d'organisation du corps, elle entre à ce titre dans un champ de préoccupations proprement anatomiques. Vésale, en accord avec Galien, considère l'anatomie comme un «savoir spéculatif»; il rappelle, dans la préface à la Fabrica, que l'anatomie est une branche de la philosophie naturelle et qu'il est nécessaire de la connaître au-delà de ses applications pratiques. Dans les représentations anatomiques, l'univers symbolique lié au corps fait surface, il participe de l'élaboration des formes propres à communiquer le savoir mixte qui caractérise la connaissance du corps humain. Selon Andrea Carlino, les significations symboliques qui structurent la représentation de l'anatomie trouvent leur expression sous la forme de métaphores:

[...] le discours anatomique [...] est en soi polymorphe, il se prête et invite à l'appropriation et à la réadaptation de son objet. Ces 
appropriations et réadaptations innombrables, attestées dans les sources, sont toujours exprimées en termes métaphoriques, en puisant aux systèmes symboliques auxquels inévitablement ce discours renvoie: en ce sens, un squelette "anatomique» n'est jamais seulement un squelette... (2001: 111)

Le pouvoir communicatif des planches anatomiques est, en ce sens, déterminé par l'association entre un discours scientifique et un discours métaphorique qui fonctionne comme un élément d'activation du parcours narratif suggéré par les images. Elles remplissent dans les traités de la Renaissance une tâche rhétorique et explicative; elles s'emploient à démontrer qu' «un savoir certain doit être extrait des matériaux de la nature» (Smith, 2004: 156; notre traduction) en légitimant l'expérience du cadavre. Le corps anatomique de la Renaissance est une réalité perméable au monde qui l'entoure. Selon les mots de Carl Havelange,

[...] la surface du corps est une porte battante s'ouvrant dans un sens et puis dans l'autre, faisant voir ainsi comme en lisière les deux réalités jumelles du microcosme et $d u$ macrocosme.

(1998: 106)

Les images du corps anatomisé révèlent cet espace liminaire de correspondances, en montrant, comme par intermittence, la structure à la fois de cet espace et de l'univers créé. Les images montrent, de manière symbolique, le corps dans sa saisie anatomique; elles tiennent lieu du corps dont elles sont les signes: «l'évidence des relations» (ibid.) que l'image met en place occulte néanmoins cette valeur de construction symbolique, en se proposant comme une irruption de données réelles qui permettent au discours sur l'anatomie de se construire. Images de l'anatomie réelle, ces illustrations se présentent en somme comme une réalité tout court.

Lillustration anatomique de la Renaissance réalise alors la rencontre de deux tensions opposées qui s'intègrent à l'appareil de persuasion construit dans l'image: la tendance à la métaphore et l'élan vers une représentation réaliste. Le pouvoir communicationnel de ces images se lie étroitement à la définition d'une "esthétique anatomique», avec ses contraintes

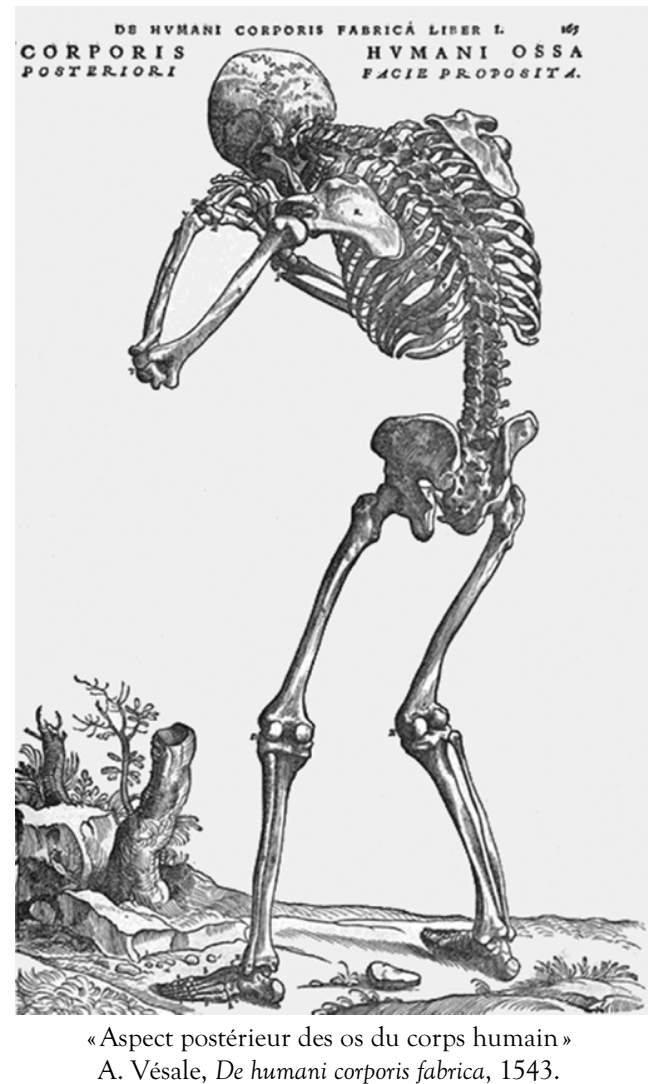

stylistiques et ses conventions. La «beauté cadavérique» des écorchés vésaliens devient une catégorie esthétique qui permet au spectateur de saisir les merveilles de l'étonnante charpente du corps humain. La notion de «fabrique» (fabrica) désigne, dans le traité intitulé De humani corporis fabrica de Vésale 3 , la "construction" en images des différents éléments qui structurent le corps. Ce mot, du latin classique, désigne l'art du faber, artisan du bronze ou du bois et plus rarement architecte, et n'est utilisé que très rarement pour désigner le corps humain; il renvoie au corps en tant que création mathématique du "Divin Architecte» tout en faisant référence à sa nature d'objet "produit» et éventuellement connaissable par l'art. La compréhension du corps suit, à rebours, le parcours de sa création: la déconstruction anatomique révèle la structure de l'homme et relève, en ce sens, d'un faire savant et créatif. 
Dans son œuvre, Vésale donne à l'iconographie une ampleur sans précédent, en exploitant pleinement les possibilités expressives offertes par le support visuel. Les trois cents planches qui illustrent la «fabrique» du corps - probablement l'œuvre de Jean de Calcar, élève du Titien - représentent une construction non seulement du corps, mais de toute une stratégie rhétorique qui en affirme le pouvoir symbolique. Le «rationalisme anatomique» de l'entreprise vésalienne entretient, en particulier, des relations profondes avec la pensée alchimique. Comme Vésale, Paracelse (1493-1541), en fondant son savoir sur l'idée d'une nature vivante, habitée par une force magique qui règle les relations entre ses éléments, «a violemment combattu la science médicale de son temps et proclamé la valeur et la nécessité de l'“expérience” (Koyré, 1971: 78). Koyré rappelle à ce propos que «la magie est naturelle parce que la nature est magique» (ibid.: 83) et que cette idée constituait un fonds commun pour les philosophes de la Renaissance. Conçu comme microcosme, le corps est ainsi soumis à l'influence des astres et se transforme à son tour, à l'instar de tous les autres éléments naturels.

La connaissance matérielle du corps fait partie du savoir alchimique. Le monde temporel, et tel l'homme qui l'habite, est "déchu»; Paracelse le qualifie de cagastrique (produit d'une corruption) en tant que provenant du péché originel qui a changé la structure de l'homme par sa rencontre même avec le monde matériel. L'état naturel plonge l'homme dans un univers en transformation dont l'alchimiste ne fait qu'imiter et accélérer les processus. De la même manière que la pierre philosophale transfigure les métaux, l'homme ne peut rejoindre son état primitif et rentrer dans l'univers céleste que grâce à une aide extérieure que Paracelse reconnaît dans le Christ, l'Homme-Dieu, qui est pour l'homme la tincture qui peut le reconduire à son état originel. Ce cadre conceptuel pose l'existence et la nécessité de l'anatomie:

Avant la chute, le corps d'Adam était un corps dynamique, il ne se nourrissait pas comme nous; il ne mangeait pas. Car bien que tout être ait besoin de "nourriture», les êtres supérieurs, les anges, par exemple, ne se nourrissent pas par la bouche comme le font les animaux et comme nous le faisons depuis. Adam n'avait pas d'intestin ni de parties sexuelles. Il n'était pas sujet à la mort. Il devait engendrer magiquement étant androgyne. (Ibid.: 107)

L'alchimie représente, en ce sens, un symbole du retour de la matière à son origine divine, de même que l'anatomie est la marque de l'insertion de l'homme dans le monde naturel. La notion de métaphore trouve alors, outre sa signification expressive, une signification méthodologique. Les «migrations métaphoriques» des significations du corps font écho à celles - métaphoriques et matérielles - qui trouvent leur actualisation dans la nature du corps et, plus généralement, dans celle de la vie qui n'est autre, elle-même, qu'un processus alchimique. Les significations allégoriques auxquelles les représentations anatomiques du corps ne semblent pas pouvoir échapper se lient ainsi aux influences philosophiques, dont l'anatomie fait l'objet, et participent, à ce titre, aux mécanismes de formation d'une pensée scientifique spécialisée dans la connaissance du corps.

Support visuel des processus d'apprentissage et de transmission du savoir qui trouvent une nouvelle actualité dans l'entreprise éditoriale de Vésale, l'image amplifie et exploite ces composantes dans la réalisation de son programme persuasif. Les éléments expressifs utilisés pour désigner le corps et le contexte qui l'entoure fonctionnent alors comme des appels: ils signalent des «bifurcations» possibles dans les sentiers interprétatifs de l'image qui, tel le corps, possède et suggère plusieurs types de lecture. On ne saurait parler du réalisme de ces images sans leur associer ce qu'Andrea Carlino définit comme un «impératif métaphorique» (1999), à savoir la nécessité de trouver des arrangements pour transmuter en curiosité scientifique le sentiment coupable de briser l'interdiction - morale plus que religieuse - de porter atteinte à l'intégrité du corps. C'est sans doute ce pourquoi le corps disséqué est, après Vésale, presque toujours représenté comme vivant, occupant comme 
tel l'espace naturel qui l'entoure. Les pages que Georges Canguilhem a écrites à ce sujet nous renseignent sur la relation que l'homme de Vésale entretient avec le monde qui l'entoure; sous un ciel «pré-copernicien» qui lui assure, microcosme dans le macrocosme, une place centrale dans la création, il s'érige comme mesure de la réalité connaissable:

Parce que le monde de Copernic commence à peine en 1543 de briller aux yeux de l'intelligence, l'homme de Vésale peut encore ignorer que sa nature de tout organique, distinct du monde quoique accordé à lui, est sur le point d'être mise en question. Elle le sera effectivement le jour où le Cosmos antique et médiéval, habitat de l'homme centré sur l'homme et comme fait pour lui, fera place à l'univers dont le centre est partout et la circonférence nulle part. (1991: 33)

Le corps humain, mis en scène dans les traités anatomiques du XVI siècle, ne se limite pas à signifier l'anatomie; il évoque aussi plusieurs métaphores qui participent à la transmission du savoir lié au corps. Son support, le livre imprimé, n'est-il pas déjà en soi un objet métaphorique?

Si le naturalisme de Paracelse invitait à feuilleter le grand «livre de la nature», l'anatomie de Vésale incitait à lire les signes de la fabrica corporis dans ce livre qui était le contexte $d u$ corps mort. En passant de ce corps mort au corps vivant, la chirurgie de Paré contribuait de son côté à l'assertion de longue date voulant que «les vrais liures soient les malades».

(Cosmacini, 2005: 262-263; notre traduction)

L'anatomie s'affirme, depuis la Renaissance, comme un modèle pour penser le corps dans sa généralité ainsi que comme un moyen pour réfléchir sur soi, en ce qu'elle participe à la construction du sujet moderne. La littérature anatomique nous renseigne sur l'incroyable intelligence formelle et fonctionnelle que l'homme découvre en lui - signe manifeste de sa nature divine mais aussi miroir de ce qu'on appelle l'âme. Objet d'une semiosis illimitée où toute signification reconduit sans cesse à une nouvelle interprétation, le corps réel est alors un signe générant à son tour d'autres signes - paroles et images constituant de possibles parcours d'interprétation et remodelant leur propre référent. Le pouvoir symbolique du corps montre ainsi la nature d'artefact du monde qui, à son tour, est et fait signe.

Représenter le corps anatomique comme un objet du monde le soumet à une objectivité qui s'informe sur le pouvoir symbolique que le corps recèle. La "fracture formelle» de l'ouverture du cadavre laisse voir le corps limité par sa peau comme un simulacre, l'incision anatomique annonce un sens ultérieur. Un soupçon de vérité, comme l'évidence soudaine d'un état de choses, surgit par la vision fragmentaire de cette chair informe, brute, qui s'offre néanmoins comme une clef de compréhension du monde.

\section{N O TES}

1. Dans le Lai d'Ignauré, l'amant de douze dames est mis à mort par les maris trompés. Ces derniers donnent son cœur et son membre viril à manger aux femmes infidèles. Cet exemple est présenté dans Le Goff et Truong (2003: 173). Pour l'approfondissement de ce thème, voir Régnier-Bohler (1979).

2. Danielle Gourevitch présente à ce sujet la figure de Giovanni Dondi, médecin et astrologue mort en 1389, qui inventa l'horloge planétaire. La question de la relation entre astrologie et technique à la fin du Moyen Âge est traitée dans White (1975).

3. Édité en 1543 chez Johannes Oporinus à Bâle, le traité de Vésale, édition en grand format, représentait un achat de coût élevé. Pour en assurer une correcte diffusion, Vésale publia en même temps les Epitomes, un résumé illustré de son œuvre majeure, en latin et en allemand. Né à Bruxelles en 1514, Vésale étudie à Louvain et Paris et complète en 1537 ses études à l'université de Padoue où, cette même année, il est nommé professeur de chirurgie et anatomie jusqu'en 1542. 


\section{RÉFÉREN CES BIBLIO G RAPH IQ U ES}

CAMPORESI, P. [(1985) 1991]: Le Officine dei sensi, Milan, Garzanti ;

[1994]: La Carne impassibile, Milan, Mondadori.

Canguilhem, G. [(1968) 1991]: L'homme de Vésale dans le monde de Copernic: 1543, Paris, Laboratoires Delagrange.

CARlinO, A. [1999]: "L'impératif métaphorique: quelques réflexions autour de l'illustration anatomique: XVe-XVIII e siècle", Traverse.

Zeitschrift für Geschichte, tome VI, fasc. 3, 23-34;

[2001]: «Entre corps et âme, ou l'espace de l'art dans

l'illustration anatomique", Littérature et médecine, ou les pouvoirs du récit, Paris, BPI/Centre Pompidou, 111.

Cocchi, A. [1745] : Discorso primo intorno all'anatomia, Florence, Gio.

Batista Zannoni, repris dans P. Camporesi ([1985] 1991).

Cosmacini, G. [(1997) 2005]: L’Arte lunga. Storia della medicina

dall'Antichità a oggi, Rome-Bari, Laterza.

GALIEN (Claudius Galenus) [1531] : De anatomicis administrationibus,

Paris, Simon de Colines.

GalimberTi, U. [(1983) 1999]: Il Corpo, Milan, Feltrinelli.

Gourevitch, A. J. [1983]: Les Catégories de la culture médiévale, Paris, Gallimard.

Havelange, C. [1998]: De l'œil et du monde. Une histoire du regard au seuil de la modernité, Paris, Fayard.

JACQUART, D. [1995]: "La scolastique médicale ", dans M. D. Grmek, Histoire de la pensée médicale en Occident I, Paris, Seuil, 175-210.

KeTHAM, J. de [1493] : Fasciculo de medicina, Venise, Johannes \&
Gregorius de Gregoriis.

KOYRÉ, A. [(1933) 1971]: Mystiques, spirituels, alchimistes du XVIe siècle allemand, Paris, Gallimard.

LE Breton, D. [(1990) 2003]: Anthropologie du corps et modernité, Paris, PUF.

LE Goff, J. et N. TRuONG [2003]: Une histoire du corps au Moyen Âge, Paris, Liana Levi.

NARDI, B. [1958]: Saggi sull'aristotelismo padovano dal secolo XIV al XVI, Florence, Sansoni.

Nutton, V. [(1995) 2003]: " Medicine in Medieval Western Europe, 1000-1500", dans L. I. Conrad et alii, The Western Medical Tradition. 800 $B C$ to $A D$ 1800, Cambridge, Cambridge University Press, 139-205. Piervirgilit, B. [1663]: Vita della B. Chiara detta della Croce da Montefalco dell'ordine di S. Agostino, Foligno, Eredi d'Agostino Alterii (repris dans P. Camporesi [1994]).

RÉGNIER-BOHLER, D. [ 1979]: Le Cour mangé. Récits érotiques et courtois des XII et XIII siècles, Paris, Stock.

SMITH, P. H. [2004]: The Body of the Artisan. Art and Experience in the Scientific Revolution, Chicago et Londres, The University of Chicago Press.

VÉSALE, A. [1543]: De humani corporis fabrica, Bâle, Johannes Oporinus. VORAGINE, J. de [2004]: La Légende dorée (Legenda Aurea), sous la dir. d'A. Boureau Paris, Gallimard.

WHITE, L. J. [1975]: «Medical Astrologers and Late Medieval Technology", Viator, nº6, 295-308. 